\title{
Copper-in-Charcoal (Cu/C) Promoted Diaryl Ether Formation
}

\author{
Bruce H. Lipshutz*, John B. Unger, and Benjamin R. Taft \\ Department of Chemistry \& Biochemistry \\ University of California \\ Santa Barbara, CA 93106
}

\section{Supporting Information}


General. Reactions were performed in oven-dried Biotage ${ }^{\circledR} 2-5 \mathrm{~mL}$ microwave vials under an argon atmosphere containing a Teflon coated stir bar and sealed with a Teflon lined septum. Solvents were freshly distilled from $\mathrm{Na}^{\circ} /$ benzophenone ketyl prior to use. $\mathrm{Cu} / \mathrm{C}$ was stored and weighed out as a black powder on the bench top. All aryl halides were obtained commercially and used as received; phenols were obtained commercially and recrystallized prior to use. All microwave experiments were performed using a Personal Chemistry (now Biotage ${ }^{\circledR}$ ) Emrys Optimizer. GC analyses were carried out using an HP-5 capillary column $(0.25 \mu \mathrm{m} \times 30 \mathrm{~m}$; cross-linked 5\% PH-ME siloxane) and a time program beginning with $5 \mathrm{~min}$ at $50{ }^{\circ} \mathrm{C}$ followed by $20^{\circ} \mathrm{C} / \mathrm{min}$ ramp to $280{ }^{\circ} \mathrm{C}$, then $20 \mathrm{~min}$ at this temp. Column chromatography was performed using Davisil Grade 633 Type 60_ silica gel. TLC analyses were performed on commercial Kieselgel $60 \mathrm{~F}_{254}$ silica gel plates. NMR spectra were obtained on Varian Inova systems using $\mathrm{CDCl}_{3}$ as solvent, with proton and carbon resonances at 400 and $100 \mathrm{MHz}$, respectively. IR spectra were obtained on an JASCO FT/IR-430 series spectrometer neat on $\mathrm{NaCl}$ plates or in a $\mathrm{KBr}$ pellet and are reported in $\mathrm{cm}^{-1}$. Mass spectral data were acquired on a VF Autospec or an analytical VG-70-250 HF instrument.

Preparation of $\mathbf{C u} / \mathbf{C}$ : Darco KB activated carbon (15.0 g, 100 mesh, $25 \% \mathrm{H}_{2} \mathrm{O}$ content) was added to a $300-\mathrm{mL}$ round-bottom flask containing a stir bar. A solution of $\mathrm{Cu}\left(\mathrm{NO}_{3}\right)_{2} \cdot 3 \mathrm{H}_{2} \mathrm{O}$ (Acros Organics, $5.00 \mathrm{~g}, 20.7 \mathrm{mmol})$ in deionized $\mathrm{H}_{2} \mathrm{O}(100 \mathrm{~mL})$ was added to activated carbon, and additional deionized $\mathrm{H}_{2} \mathrm{O}(125 \mathrm{~mL})$ was added to wash down the sides of the flask. The flask was purged under argon and stirred vigorously for $30 \mathrm{~min}$. The flask was submerged in an ultrasonic bath under a positive argon flow for 1 hour. The flask was attached to an argon-purged distillation setup and placed in a preheated $175-180{ }^{\circ} \mathrm{C}$ sand bath with stirring plate. As the distillation ended, the flask temperature began to rise and was held below $210{ }^{\circ} \mathrm{C}$ for an additional $15 \mathrm{~min}$. Upon cooling to rt, toluene $(75 \mathrm{~mL})$ was added to wash down the sides of the flask. The flask was again placed into a hot sand bath until the toluene $/ \mathrm{H}_{2} \mathrm{O}$ azeotrope had distilled. Once the distillation was finished, the azeotropic distillation was repeated a second time. Upon cooling to room temperature, the black solid was washed with toluene (2_50 mL) under argon into a predried 150-mL coarse-fritted funnel (in vacuo). The fritted funnel was turned upside down under vacuum for $5 \mathrm{~h}$ until the $\mathrm{Cu} / \mathrm{C}$ fell from the frit into the collection flask. The collection flask was then heated in vacuo in a $110-115^{\circ} \mathrm{C}$ sand bath for $18 \mathrm{~h}$ to further 
dry the catalyst. The impregnated charcoal ( $c a .13 \mathrm{~g})$ was transferred to, and stored in, an amber vial.

Simplified Preparation of Cu/C: Darco KB activated carbon $\left(50.0\right.$ g, 100 mesh, $25 \% \mathrm{H}_{2} \mathrm{O}$ content) was added to a $500-\mathrm{mL}$ round-bottom flask containing a stir bar. A solution of $\mathrm{Cu}\left(\mathrm{NO}_{3}\right)_{2} \cdot 3 \mathrm{H}_{2} \mathrm{O}$ (Acros Organics, $11.114 \mathrm{~g}, 46.0 \mathrm{mmol}$ ) in deionized $\mathrm{H}_{2} \mathrm{O}(100 \mathrm{~mL}$ ) was added to the flask and deionized $\mathrm{H}_{2} \mathrm{O}(100 \mathrm{~mL})$ was further added to wash down the sides of the flask. The flask was loosely capped and stirred under air for $30 \mathrm{~min}$, then submerged in an ultrasonic bath for $7 \mathrm{~h}$. Subsequent washing with toluene $(2 \times 50 \mathrm{~mL})$ and air drying $(3 \mathrm{~h})$ by vacuum filtration yielded ca. 85 grams of "wet" $\mathrm{Cu} / \mathrm{C}$. The catalyst was then further dried in vacuo at $120^{\circ} \mathrm{C}$ overnight to yield 41 grams of "dry" $\mathrm{Cu} / \mathrm{C}$. The impregnated charcoal was transferred to, and stored in, an amber bottle.

\section{Representative procedure for the microwave assisted $\mathrm{Cu} / \mathrm{C}$-catalyzed Ullmann aryl ether} coupling; compound 2, Scheme 2. $\mathrm{Cu} / \mathrm{C}(30 \mathrm{mg}, 0.05 \mathrm{mmol}, 1.8 \mathrm{mmol} / \mathrm{g}), 1,10$-phenanthroline (90 mg, $0.50 \mathrm{mmol})$, cesium carbonate $(390 \mathrm{mg}, 1.2 \mathrm{mmol})$, and estrone (271 $\mathrm{mg}, 1.0 \mathrm{mmol})$ were added to a oven-dried Biotage ${ }^{\circledR} 2-5 \mathrm{~mL}$ process vial under argon at $\mathrm{rt}$. Dry dioxane $(2.0$ $\mathrm{mL}$ ) was added by syringe and the slurry allowed to stir at $\mathrm{rt}$ for $30 \mathrm{~min}$. 3-Bromopyridine $(0.145 \mathrm{~mL}, 1.5 \mathrm{mmol})$ was added via micro-syringe and the resulting heterogeneous mixture was heated in the microwave with the following settings; Temperature: $220^{\circ} \mathrm{C}$, Time: $1800 \mathrm{sec}$., Fixed hold time: On, Sample Absorption: Normal, Pre-stirring: $10 \mathrm{sec}$. After cooling to rt, the crude reaction mixture was filtered through Celite ${ }^{\circledR}$ and the filter cake was further rinsed with ethyl acetate. Volatiles were removed on a rotary evaporator and the crude product was then purified by flash chromatography on silica gel, (3/2 Hexanes/Ethyl Acetate) $R_{f}=0.25$, to yield $299 \mathrm{mg}(86 \%)$ as a colorless solid. 
Compound Characterization Data. Aryl ethers: in Table 1, and Table 2 entries 1 and 5, are known compounds; spectral data correspond to that in the literature. ${ }^{[1]}$

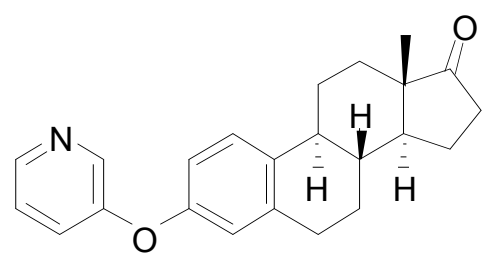

(Scheme 2, compound 2). Colorless amorphous solid, $\mathrm{mp}=146-147{ }^{\circ} \mathrm{C} .{ }^{1} \mathrm{H}$ NMR $(400 \mathrm{MHz}$, $\left.\mathrm{CDCl}_{3}\right) \delta 8.39-8.30(\mathrm{~m}, 2 \mathrm{H}), 7.29-7.19(\mathrm{~m}, 3 \mathrm{H}), 6.77(\mathrm{dd}, J=2.8,8.8 \mathrm{~Hz}, 1 \mathrm{H}), 6.72(\mathrm{~s}, 1 \mathrm{H})$, 2.89-2.81 (m, 2H), $2.47(\mathrm{dd}, J=19.2,8.8 \mathrm{~Hz}, 1 \mathrm{H}), 2.42-1.89(\mathrm{~m}, 6 \mathrm{H}), 1.65-1.35(\mathrm{~m}, 6 \mathrm{H}), 0.88$ $(\mathrm{s}, 3 \mathrm{H}) .{ }^{13} \mathrm{C} \mathrm{NMR}\left(100 \mathrm{MHz}, \mathrm{CDCl}_{3}\right) \delta 220.9,154.2,144.2,141.4,138.7,135.8,127.0,125.3$, $124.1,119.2,116.5,50.5,48.1,44.2,38.2,35.9,31.6,29.6,26.5,25.9,21.7,13.9$. IR $(\mathrm{KBr}$ pellet): 3074, 3044, 2932, 2888, 2854, 1736, 1605, 1570, 1492, 1471, 1422, 1276, 1259, 1228, $1187 \mathrm{~cm}^{-1}$. MS (EI): $m / z(\%): 348(25), 347(100), 290(11), 223(11), 128(10), 97(10)$. HREIMS calcd. for $\mathrm{C}_{23} \mathrm{H}_{25} \mathrm{NO}_{2}\left[\mathrm{M}^{+}\right]=347.1885$, found 347.1870 .

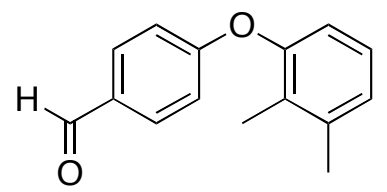

(Table 2, entry 2). Colorless oil. ${ }^{1} \mathrm{H}$ NMR (400 MHz, $\left.\mathrm{CDCl}_{3}\right) \delta 9.91(\mathrm{~s}, 1 \mathrm{H}), 7.83(\mathrm{~m}, 2 \mathrm{H})$, $7.15(\mathrm{t}, J=8.0 \mathrm{~Hz}, 1 \mathrm{H}), 7.08(\mathrm{dt}, J=0.4,7.2 \mathrm{~Hz}, 1 \mathrm{H}), 6.96(\mathrm{~m}, 2 \mathrm{H}), 6.88(\mathrm{dt}, 0.4,8.0 \mathrm{~Hz}, 1 \mathrm{H})$, $2.34(\mathrm{~s}, 3 \mathrm{H}), 2.10(\mathrm{~s}, 3 \mathrm{H}) .{ }^{13} \mathrm{C} \mathrm{NMR}\left(100 \mathrm{MHz}, \mathrm{CDCl}_{3}\right) \delta 191.0,163.9,152.7,139.6,132.2$, 130.9, 129.4, 127.2, 126.9, 119.0, 116.5, 20.3, 12.4. IR (neat NaCl plate): 3054, 3023, 2921, 2827, 2734, 1696, 1602, 1468, 1246, 1155, 1062, $834 \mathrm{~cm}^{-1}$. MS (EI): $m / z(\%): 226(100), 197$ (7), 105(20), 77(16), 51(6). HREIMS calcd. for $\mathrm{C}_{15} \mathrm{H}_{14} \mathrm{O}_{2} \mathrm{Na}[\mathrm{M}+\mathrm{Na}]=249.0891$, found 249.0897. 


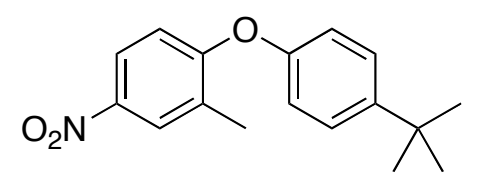

(Table 2, entry 3). Colorless oil. ${ }^{1} \mathrm{H}$ NMR $\left(400 \mathrm{MHz}, \mathrm{CDCl}_{3}\right) \delta 7.88(\mathrm{dd}, J=2.4,8.4 \mathrm{~Hz}, 1 \mathrm{H})$, $7.66(\mathrm{~d}, J=2.4 \mathrm{~Hz}, 1 \mathrm{H}), 7.40(\mathrm{~m}, 3 \mathrm{H}), 6.94(\mathrm{~m}, 2 \mathrm{H}), 2.41$ (s, 3H), $1.35(\mathrm{~s}, 9 \mathrm{H}) .{ }^{13} \mathrm{C}$ NMR $(100$ $\left.\mathrm{MHz}, \mathrm{CDCl}_{3}\right) \delta$ 156.4, 153.7, 147.4, 137.2, 131.6, 127.2, 118.6, 118.0, 112.4, 34.6, 31.7, 16.9. IR (neat $\mathrm{NaCl}$ plate): $3039,2963,2868,1600,1522,1347,1239,833,741 \mathrm{~cm}^{-1}$. MS (EI): $\mathrm{m} / \mathrm{z}$ (\%): 285(22), 270(100), 224(5), 97(7). HREIMS calcd. for $\mathrm{C}_{17} \mathrm{H}_{19} \mathrm{NO}_{3}\left[\mathrm{M}^{+}\right]=285.1365$, found 285.1372 .

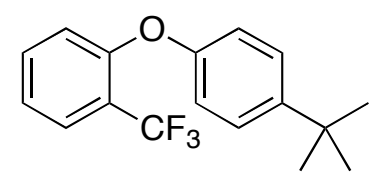

(Table 2, entry 4). Colorless oil. ${ }^{1} \mathrm{H}$ NMR $\left(400 \mathrm{MHz}, \mathrm{CDCl}_{3}\right) \delta 7.70(\mathrm{dd}, J=1.2,7.6 \mathrm{~Hz}, 1 \mathrm{H})$, $7.44(\mathrm{dt}, J=1.2,7.2 \mathrm{~Hz} 1 \mathrm{H}), 7.39$ (m, 2H), 7.15 (t, $J=7.6 \mathrm{~Hz}, 1 \mathrm{H}), 6.99(\mathrm{~m}, 2 \mathrm{H}), 6.95$ (d, $J=$ $8.4 \mathrm{~Hz}, 1 \mathrm{H}), 1.344(\mathrm{~s}, 9 \mathrm{H}) .{ }^{13} \mathrm{C} \mathrm{NMR}\left(100 \mathrm{MHz}, \mathrm{CDCl}_{3}\right) \delta$ 154.1, 147.3, 133.4, 127.3, 127.3, 127.0, 122.5, 119.3, 118.9, 34.6, 31.7. IR (neat $\mathrm{NaCl}$ plate): 3042, 2965, 2870, 1609, 1491, 1322, 1248, 1137, $759 \mathrm{~cm}^{-1}$. MS (EI): $\mathrm{m} / \mathrm{z}$ (\%): 294(23), 279(100), 251(5), 199(5), 125(5), 118 (5), 57(5). HREIMS calcd. for $\mathrm{C}_{17} \mathrm{H}_{17} \mathrm{~F}_{3} \mathrm{O}\left[\mathrm{M}^{+}\right]=294.1231$, found 294.1243 .

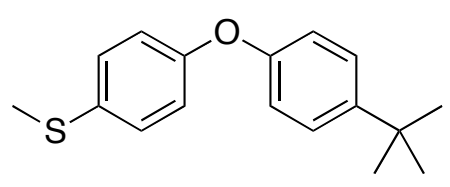

(Table 2, entry 6). Colorless oil. ${ }^{1} \mathrm{H}$ NMR $\left(400 \mathrm{MHz}, \mathrm{CDCl}_{3}\right) \delta 7.35(\mathrm{~m}, 2 \mathrm{H}), 7.27$ (m, 2H), 6.95 (m, 2H), 2.49 (s, 3H), 1.33 (s, 9H). ${ }^{13} \mathrm{C}$ NMR (100 MHz, $\left.\mathrm{CDCl}_{3}\right)$ \& 155.9, 154.9, 146.4, 132.0, 129.4, 126.8, 119.6, 118.4, 34.5, 31.7, 17.5. IR (neat NaCl plate): 3042, 2962, 2868, 1588, 1487, 1363, 1240, 1012, $827 \mathrm{~cm}^{-1}$. MS (EI): $\mathrm{m} / z$ (\%): 272(78), 257(100), 139(17), 129(6), 115(15), 91(5), 77(7). HREIMS calcd. for $\mathrm{C}_{17} \mathrm{H}_{20} \mathrm{OS}\left[\mathrm{M}^{+}\right]=272.1235$, found 272.1232. 


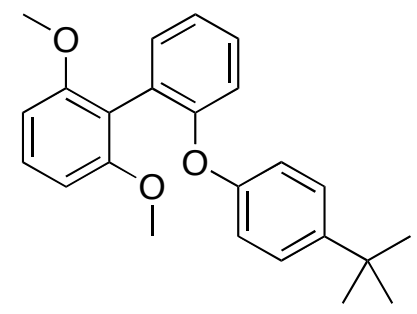

(Table 2, entry 7). Colorless needlelike crystals, $m p=142-143{ }^{\circ} \mathrm{C} .{ }^{1} \mathrm{H}$ NMR $(400 \mathrm{MHz}$, $\left.\mathrm{CDCl}_{3}\right) \delta$ 7.33-7.22 (m, 5H), 7.18 (dt, $\left.J=1.2,7.2 \mathrm{~Hz}, 1 \mathrm{H}\right), 7.02(\mathrm{~m}, 1 \mathrm{H}), 6.88(\mathrm{~m}, 2 \mathrm{H}), 6.58(\mathrm{~d}, J$ $=8.4 \mathrm{~Hz}, 2 \mathrm{H}), 3.68(\mathrm{~s}, 6 \mathrm{H}), 1.30(\mathrm{~s}, 9 \mathrm{H}) .{ }^{13} \mathrm{C} \mathrm{NMR}\left(100 \mathrm{MHz}, \mathrm{CDCl}_{3}\right) \delta 158.2,155.8,145.3$, 133.0, 129.0, 128.6, 126.4, 126.1, 122.9, 119.2, 118.4, 104.0, 55.9, 31.7. IR (KBr pellet): 3064, 2962, 2835, 1590, 1505, 1470, 1249, 1109, $730 \mathrm{~cm}^{-1}$. MS (EI): $\mathrm{m} / z$ (\%): 362(81), 347(100), 214 (7), 198(17), 183(11), 174(5), 155(12), 91(5), 57(5). HREIMS calcd. for $\mathrm{C}_{24} \mathrm{H}_{26} \mathrm{O}_{3}\left[\mathrm{M}^{+}\right]=$ 362.1882 , found 362.1894 .

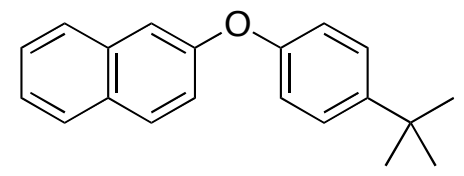

(Table 2, entry 8). Colorless solid, $\mathrm{mp}=68-69{ }^{\circ} \mathrm{C} .{ }^{1} \mathrm{H}$ NMR $\left(400 \mathrm{MHz}, \mathrm{CDCl}_{3}\right) \delta 7.84(\mathrm{~m}$, 2H), 7.72 (dd, $J=0.4,8.0 \mathrm{~Hz}, 1 \mathrm{H}), 7.48-7.37$ (m, 4H), 7.30 (m, 2H), 7.03 (m, 2H), 1.36 (s, 9H). ${ }^{13} \mathrm{C}$ NMR $\left(100 \mathrm{MHz}, \mathrm{CDCl}_{3}\right) \delta 155.6,154.8,146.5,134.5,130.2,130.0,127.9,127.3,126.8$, 126.6, 124.8, 120.2, 118.8, 113.9, 34.6, 31.7. IR (neat NaCl plate): 3057, 2962, 2904, 2868, 1631, 1597, 1505, 1464, 1251, 1176, 963, 842, $750 \mathrm{~cm}^{-1}$. MS (EI): $\mathrm{m} / z$ (\%): 276(44), 261(100), 130(6), 127(10), 116(17), 115(20), 77(5). HREIMS calcd. for $\mathrm{C}_{20} \mathrm{H}_{20} \mathrm{O}\left[\mathrm{M}^{+}\right]=276.1514$, found 276.1517.

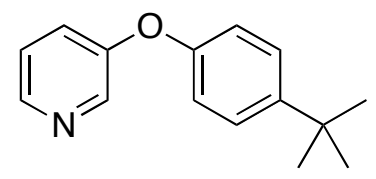

(Table 2, entry 9). Colorless oil. ${ }^{1} \mathrm{H}$ NMR (400 MHz, $\left.\mathrm{CDCl}_{3}\right) \delta 8.41$ (d, $\left.J=2.4 \mathrm{~Hz}, 1 \mathrm{H}\right), 8.35$ (dd, $J=1.6,4.4 \mathrm{~Hz}, 1 \mathrm{H}), 7.39(\mathrm{~m}, 2 \mathrm{H}), 7.31-7.23(\mathrm{~m}, 2 \mathrm{H}), 6.97(\mathrm{~m}, 2 \mathrm{H}), 1.34(\mathrm{~s}, 9 \mathrm{H}) .{ }^{13} \mathrm{C}$ NMR $\left(100 \mathrm{MHz}, \mathrm{CDCl}_{3}\right) \delta 154.5,154.0,147.3,144.2,141.4,127.1,125.3,124.2,118.8,34.6,31.7$. 
IR (neat NaCl plate): 3041, 2962, 1574, 1508, 1474, 1422, 1248, $707 \mathrm{~cm}^{-1}$. MS (EI): $m / z(\%)$ : 227(29), 212(100), 184(6), 172(5), 118(6), 91(5), 78(8), 51(7). HREIMS calcd. for $\mathrm{C}_{15} \mathrm{H}_{17} \mathrm{NO}$ $\left[\mathrm{M}^{+}\right]=227.1310$, found 227.1308 .

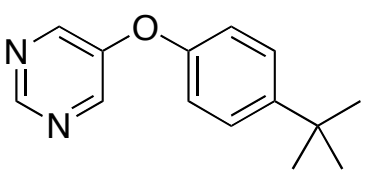

(Table 2, entry 10). Colorless oil. ${ }^{1} \mathrm{H}$ NMR $\left(400 \mathrm{MHz}, \mathrm{CDCl}_{3}\right) \delta 8.94$ (s, 1H), $8.46(\mathrm{~s}, 2 \mathrm{H})$, $7.41(\mathrm{~m}, 2 \mathrm{H}), 6.99$ (m, 2H), 1.33 (s, 9H). ${ }^{13} \mathrm{C} \mathrm{NMR}\left(100 \mathrm{MHz}, \mathrm{CDCl}_{3}\right) \delta$ 153.1, 152.9, 152.8, 148.3, 146.9, 127.3, 118.9, 34.6, 31.6. IR (neat $\mathrm{NaCl}$ plate): 3039, 2963, 2869, 1560, 1508, 1408, 1261, 1174, 1014, 837, 721, $616 \mathrm{~cm}^{-1}$. MS (EI): $\mathrm{m} / \mathrm{z}(\%): 228(33), 213(100), 185(11)$, 173 (5), 118(7). HREIMS calcd. for $\mathrm{C}_{14} \mathrm{H}_{16} \mathrm{~N}_{2} \mathrm{O}\left[\mathrm{M}^{+}\right]=228.1263$, found 228.1258 .

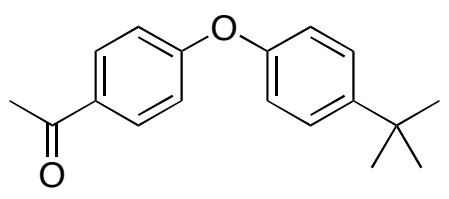

(Scheme 3). Colorless oil. ${ }^{1} \mathrm{H}$ NMR (400 MHz, $\left.\mathrm{CDCl}_{3}\right) \delta 7.94(\mathrm{~m}, 2 \mathrm{H}), 7.41(\mathrm{~m}, 2 \mathrm{H}), 7.00(\mathrm{~m}$, 4H), $2.58(\mathrm{~s}, 3 \mathrm{H}), 1.35(\mathrm{~s}, 9 \mathrm{H}) .{ }^{13} \mathrm{C} \mathrm{NMR}\left(100 \mathrm{MHz}, \mathrm{CDCl}_{3}\right) \delta$ 197.0, 162.5, 153.1, 147.8, 131.8, 130.8, 127.1, 119.9, 117.2, 34.7, 31.7, 26.7. IR (neat NaCl plate): 3043, 2963, 2871, 1682, 1592, 1500, 1244, 1172, $835 \mathrm{~cm}^{-1}$. MS (EI): $m / z$ (\%): 268(29), 253(100), 117(6), 105(7), 91(6), 77(6), 43(21). HREIMS calcd. for $\mathrm{C}_{18} \mathrm{H}_{20} \mathrm{O}_{2}\left[\mathrm{M}^{+}\right]=268.1463$, found 268.1471 .

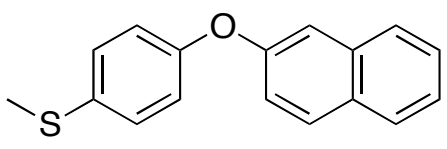

(Table 3, entry 4). Colorless solid, $\mathrm{mp}=53-54{ }^{\circ} \mathrm{C} .{ }^{1} \mathrm{H}$ NMR $\left(400 \mathrm{MHz}, \mathrm{CDCl}_{3}\right) \delta 7.83(\mathrm{~m}$, 2H), $7.70(\mathrm{dd}, J=0.8,8.0 \mathrm{~Hz}, 1 \mathrm{H}), 7.44(\mathrm{~m}, 2 \mathrm{H}), 7.32-7.24(\mathrm{~m}, 4 \mathrm{H}), 7.03(\mathrm{~m}, 2 \mathrm{H}), 2.50(\mathrm{~s}, 3 \mathrm{H})$. ${ }^{13} \mathrm{C}$ NMR $\left(100 \mathrm{MHz}, \mathrm{CDCl}_{3}\right) \delta 155.3,155.3,134.5,132.8,130.3,130.1,129.3,127.9,127.3$, 126.8, 124.9, 120.1, 120.0, 113.9, 17.2. IR (neat $\mathrm{NaCl}$ plate): 3056, 2919, 1631, 1587, 1487, 
1356, 1250, 1170, 963, 812, $750 \mathrm{~cm}^{-1}$. MS (EI): $\mathrm{m} / z$ (\%): 266(100), 223(10), 191(8), 179(5), 127 (16). HREIMS calcd. for $\mathrm{C}_{17} \mathrm{H}_{14} \mathrm{OS}\left[\mathrm{M}^{+}\right]=266.0765$, found 266.0760 .

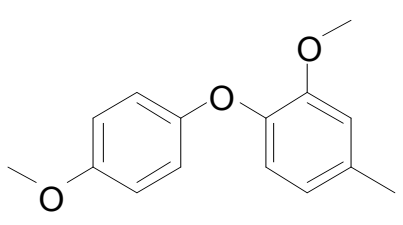

(Table 3, entry 5). Colorless waxy solid, $\mathrm{mp}=39-40{ }^{\circ} \mathrm{C} .{ }^{1} \mathrm{H}$ NMR $\left(400 \mathrm{MHz}, \mathrm{CDCl}_{3}\right) \delta$ б 6.95 $6.91(\mathrm{~m}, 2 \mathrm{H}), 6.87-6.78(\mathrm{~m}, 4 \mathrm{H}), 6.69(\mathrm{~d}, J=8.0 \mathrm{~Hz}, 1 \mathrm{H}), 3.86(\mathrm{~s}, 3 \mathrm{H}), 3.79(\mathrm{~s}, 3 \mathrm{H}), 2.36(\mathrm{~s}$, $3 \mathrm{H}) .{ }^{13} \mathrm{C}$ NMR $\left(100 \mathrm{MHz}, \mathrm{CDCl}_{3}\right)$ _ 155.2, 151.6, 150.7, 144.1, 134.0, 121.4, 119.6, 118.9, 114.7, 113.6, 56.0, 55.7, 21.4. IR (neat $\mathrm{NaCl}$ plate): 3044, 3000, 2935, 2833, 1593, 1500, 1463 , 1410, 1285, 1220, 1152, 1125, 1102, $1036 \mathrm{~cm}^{-1}$. MS (EI): $\mathrm{m} / z$ (\%): 245(16), 244(100), 229(12). HREIMS calcd. for $\mathrm{C}_{15} \mathrm{H}_{16} \mathrm{O}_{3}\left[\mathrm{M}^{+}\right]=244.1099$, found 244.1105.

\section{References:}

(1) (a) Samuelson, A. G.; Ghosh, R. New J. Chem. 2004, 28, 1390. (b) Reider, P.; Volante, R. P.; Dormer, P. G.; Tschaen, D.; Song, J. Z.; Buck, E. Org. Lett. 2002, 4, 1623. (c) Ma, D.; Cai, Q. Org. Lett. 2003, 5, 3799. 

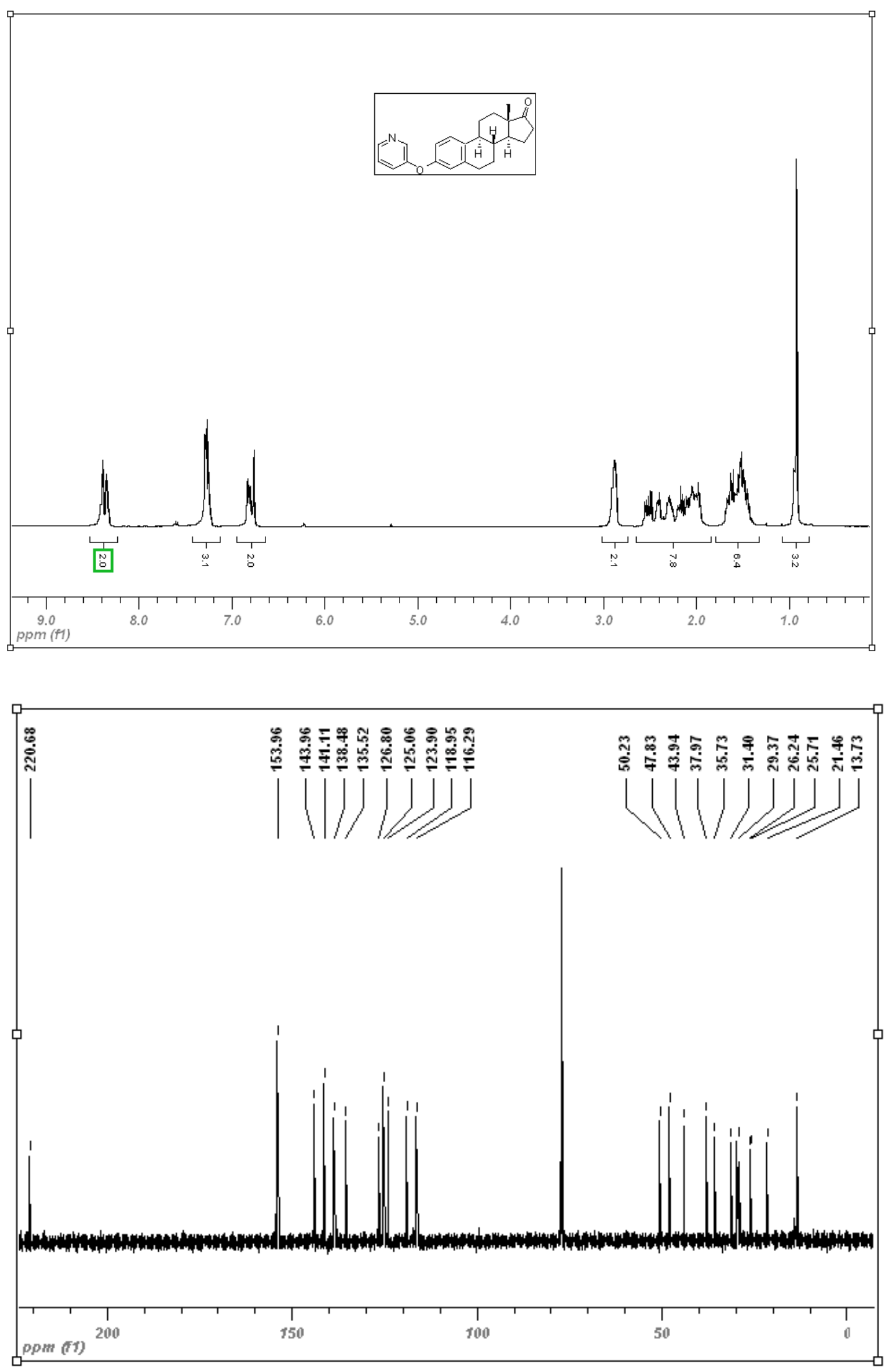
<smiles>Cc1cccc(Oc2ccc(C=O)cc2)c1C</smiles>
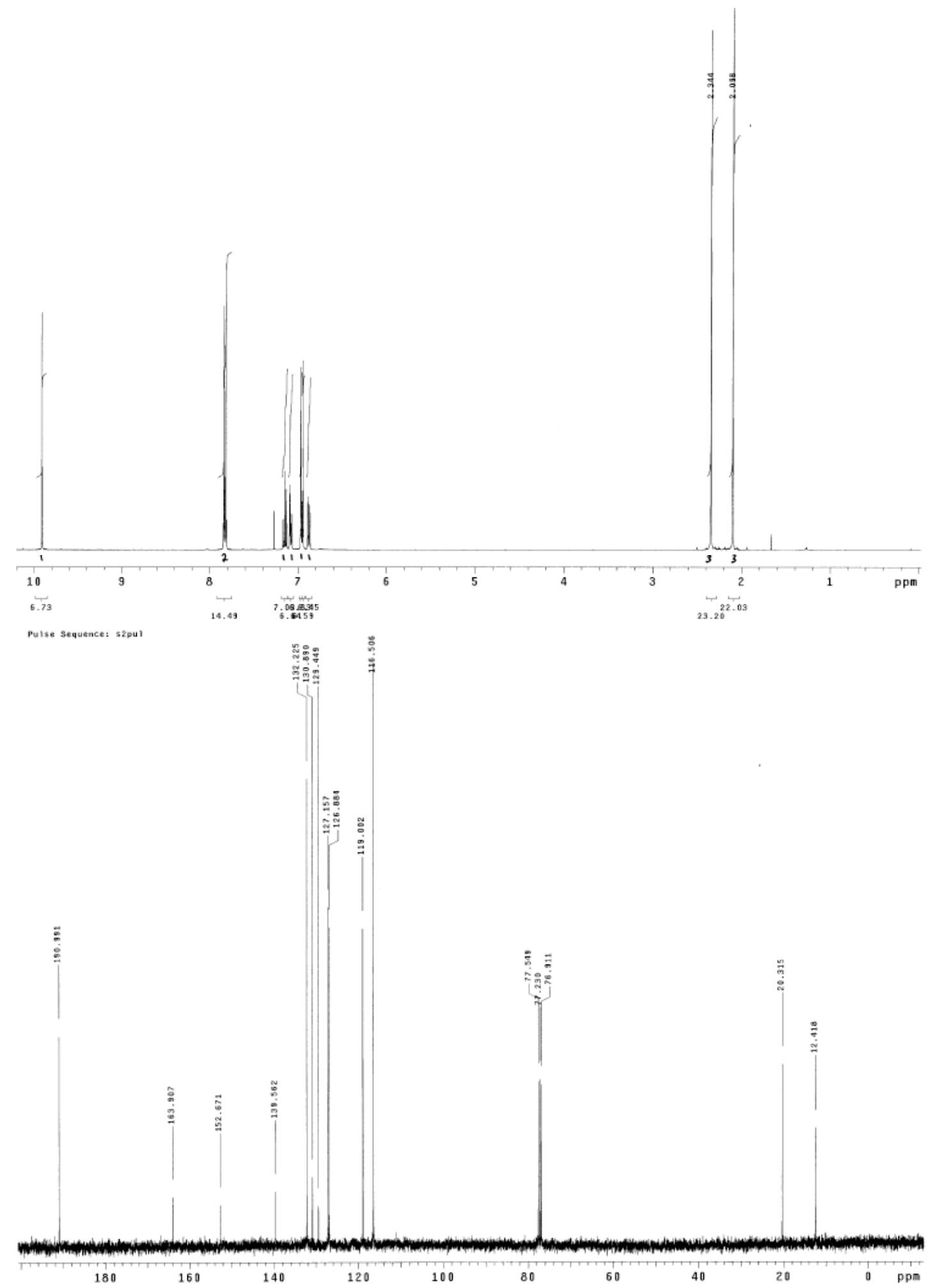

SI-10 

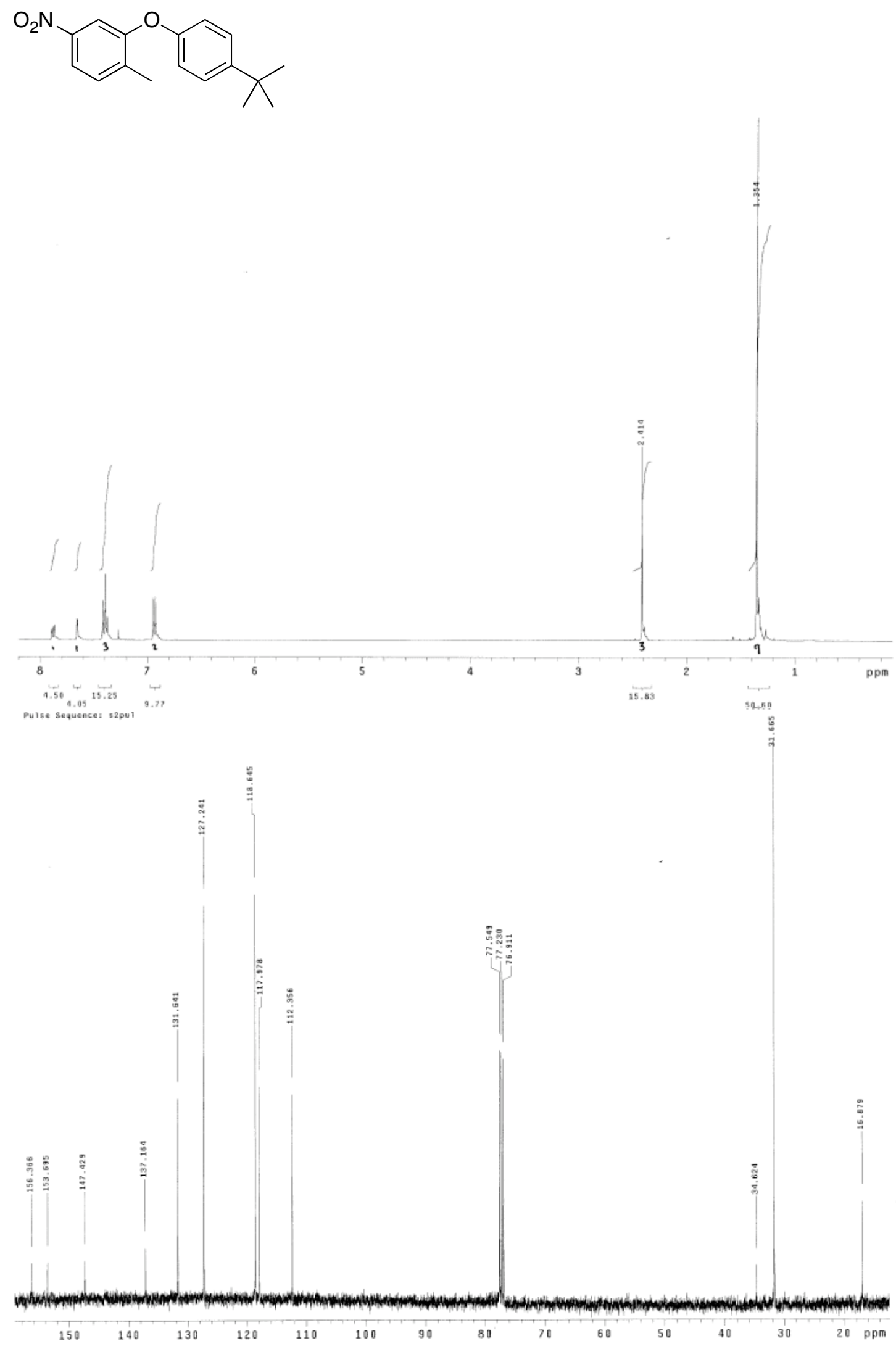

SI-11 

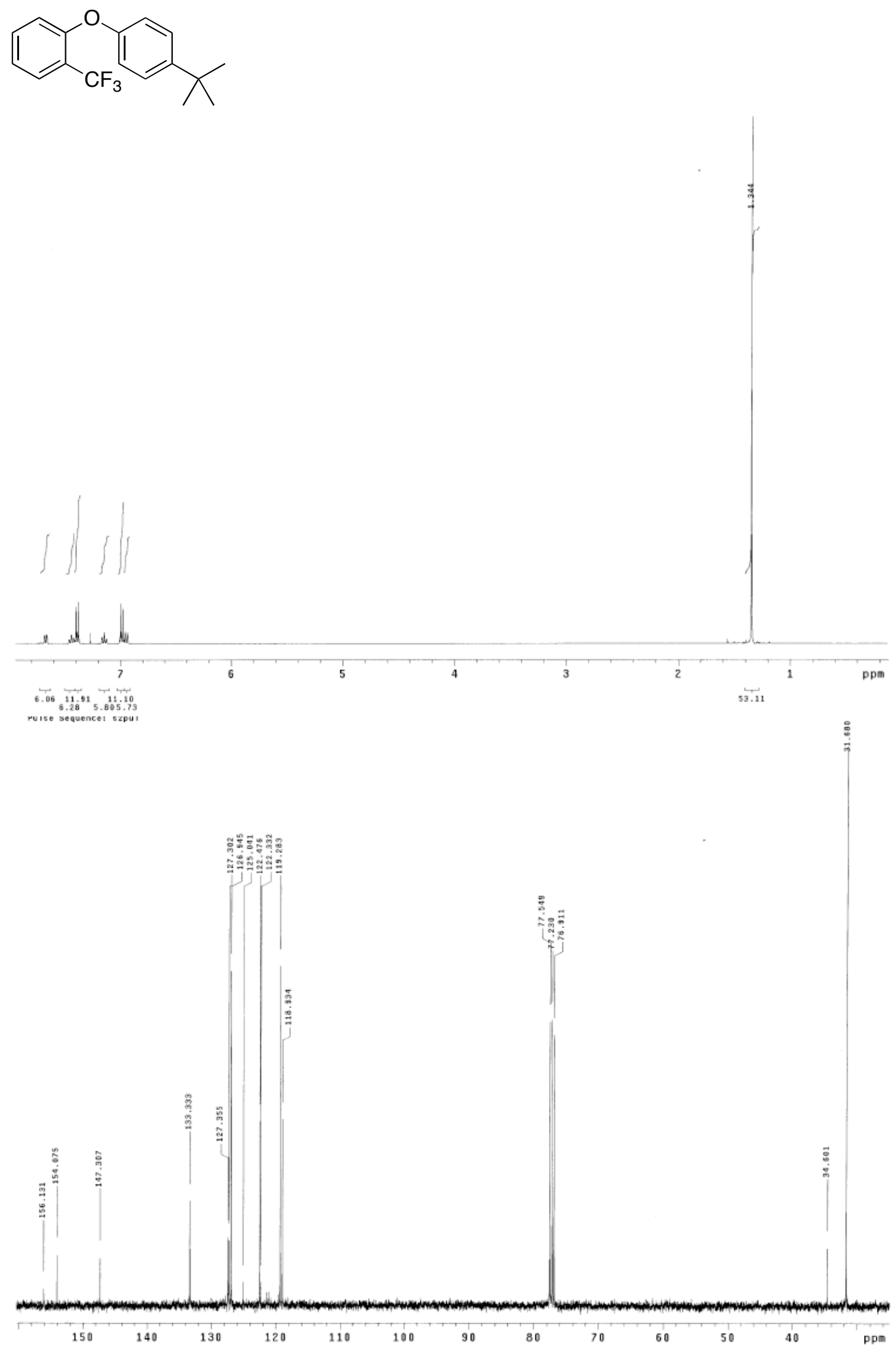

SI-1? 

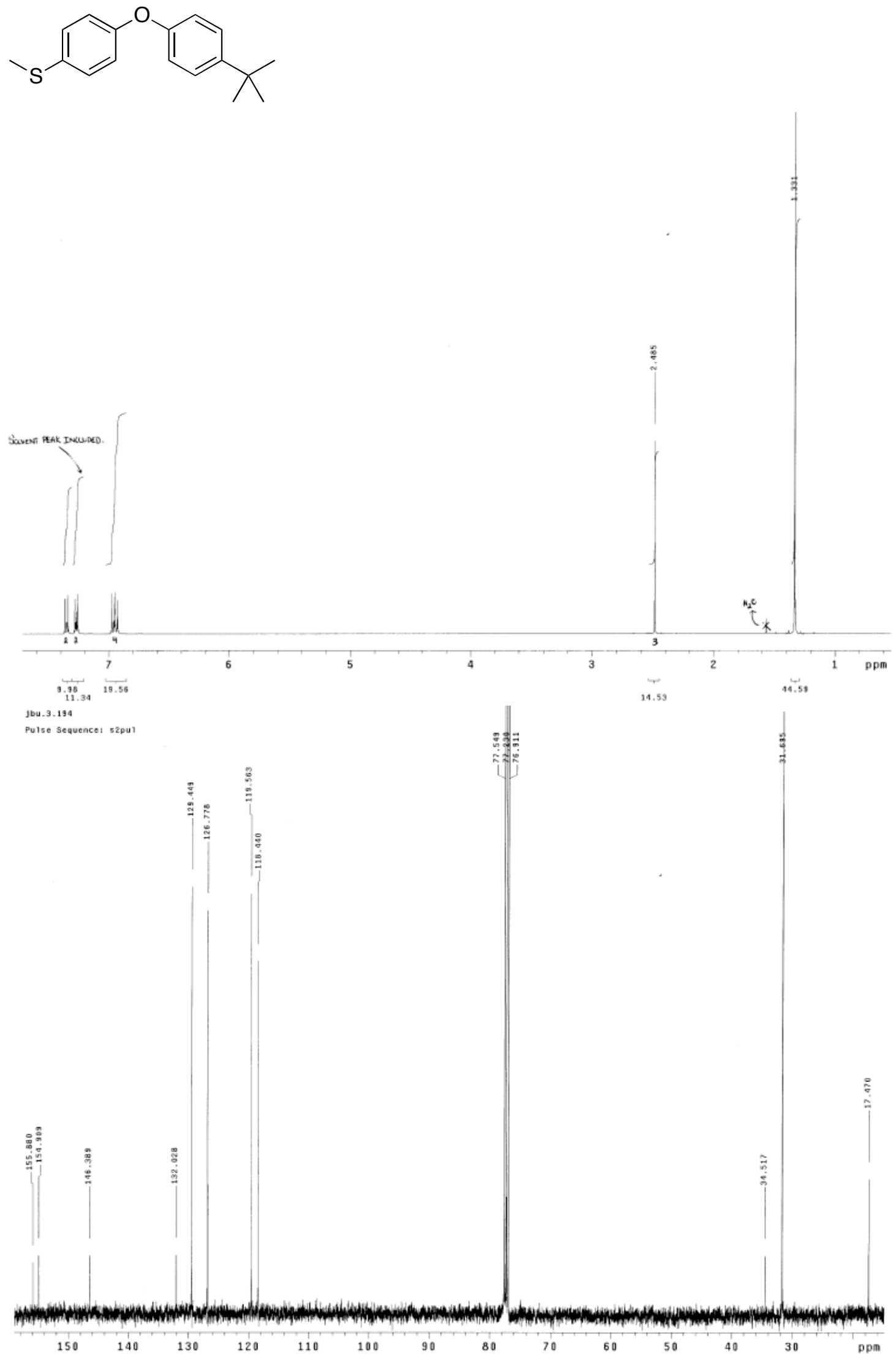

SI-13 
<smiles>COc1cccc(OC)c1-c1ccccc1Oc1ccc(C(C)(C)C)cc1</smiles>
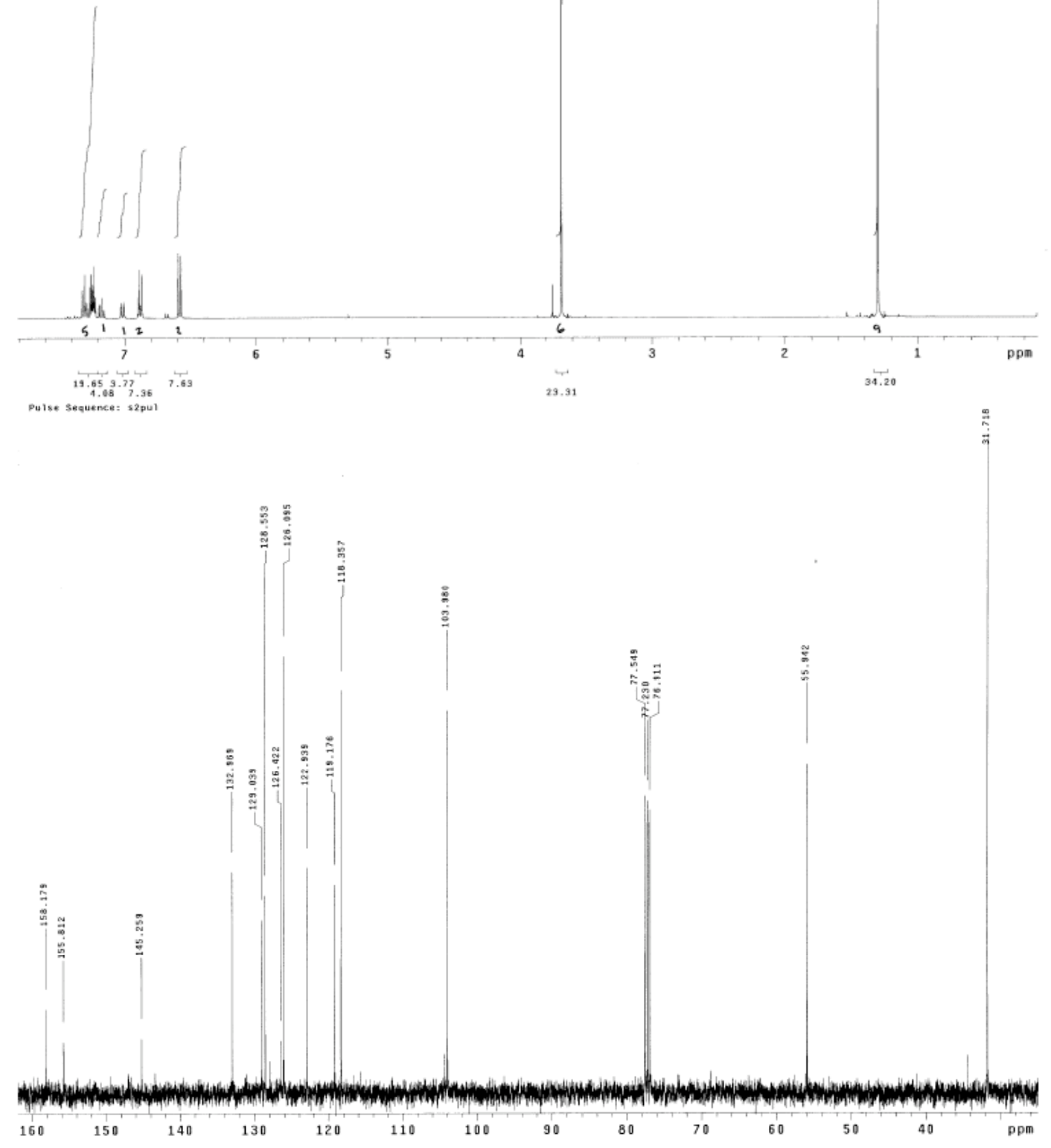

SI-14 
<smiles>CC(C)(N)c1ccc(Oc2ccc3ccccc3c2)cc1</smiles>

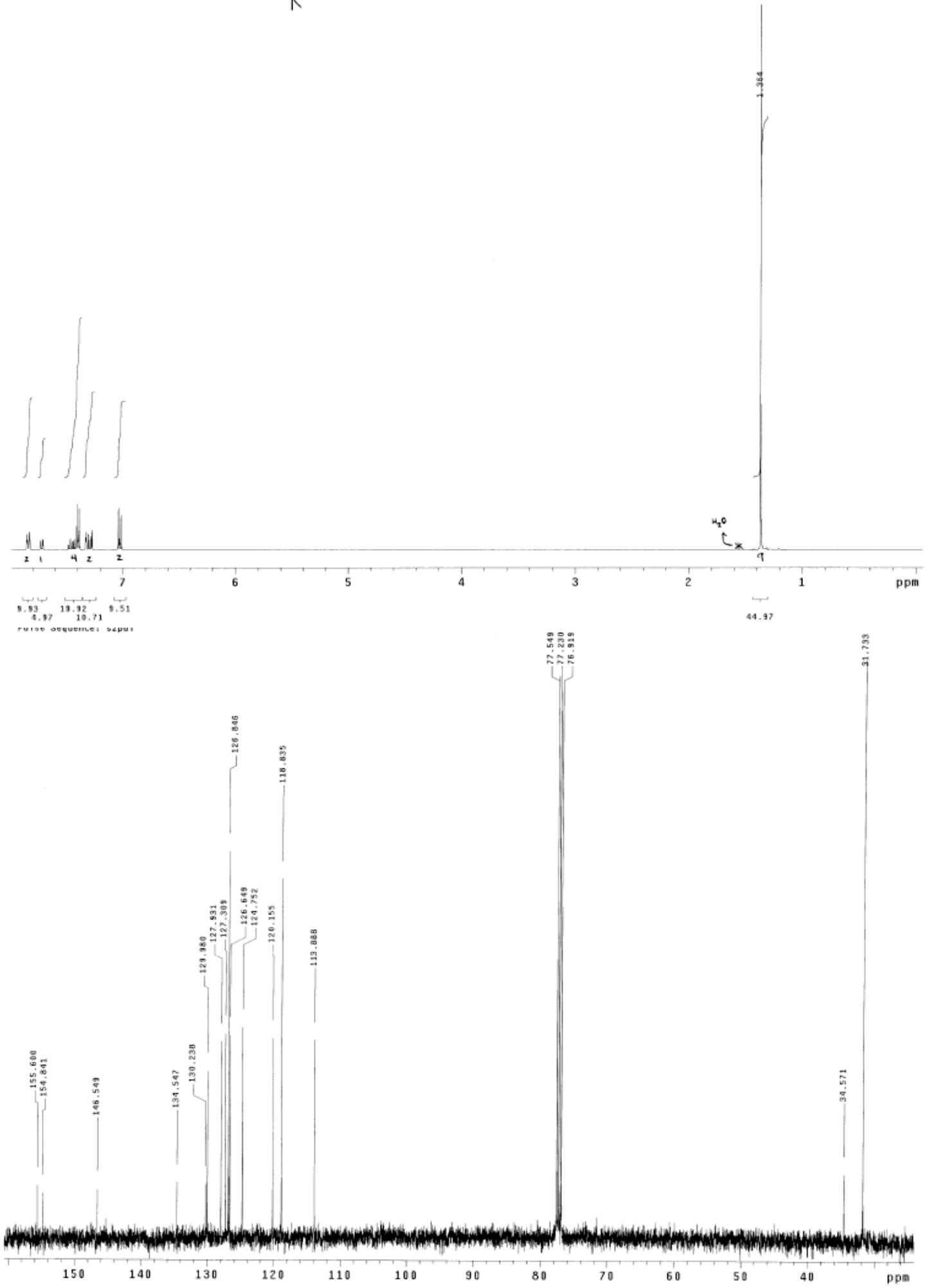

SI-15 


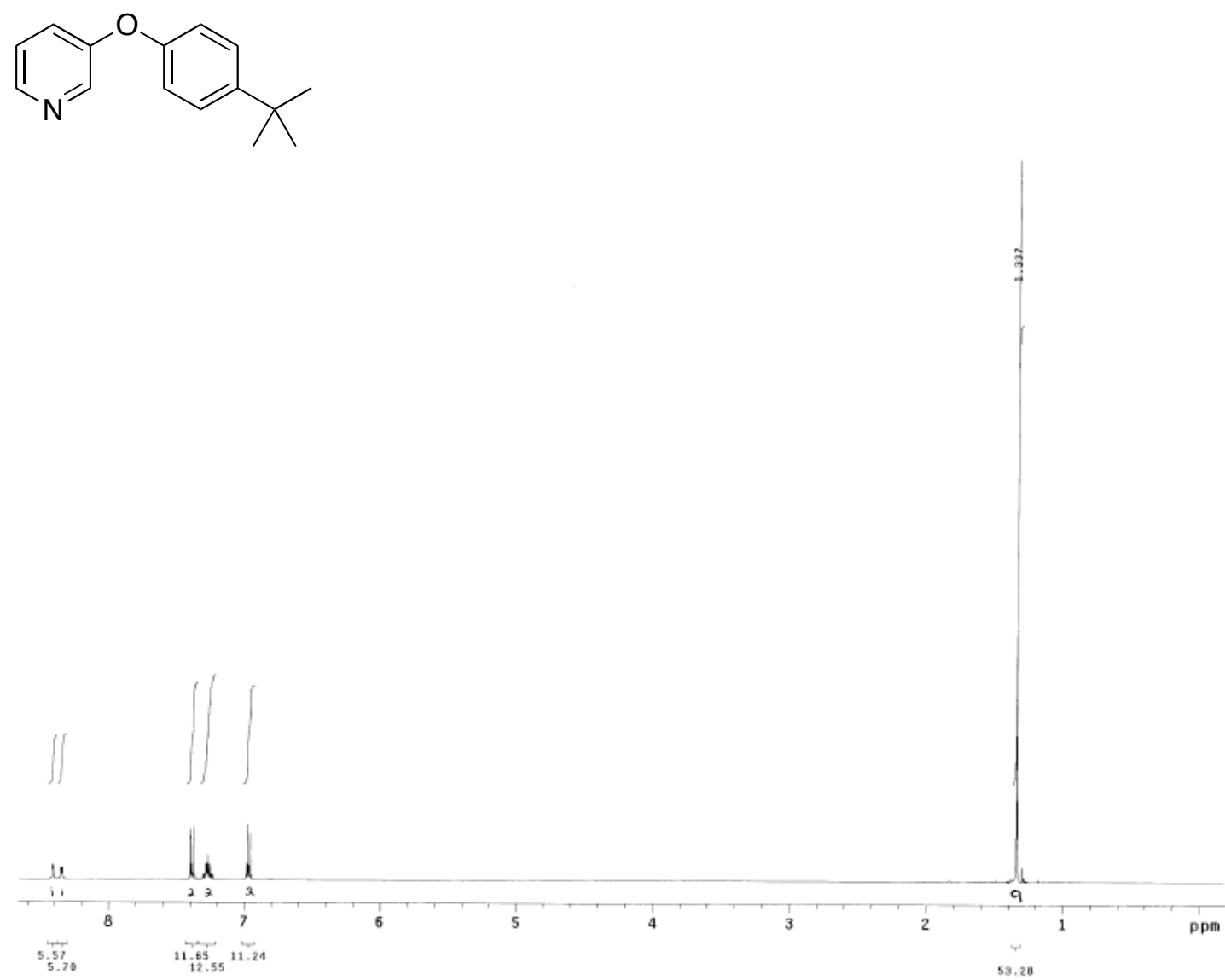

Pulse Sequence: 52 pul

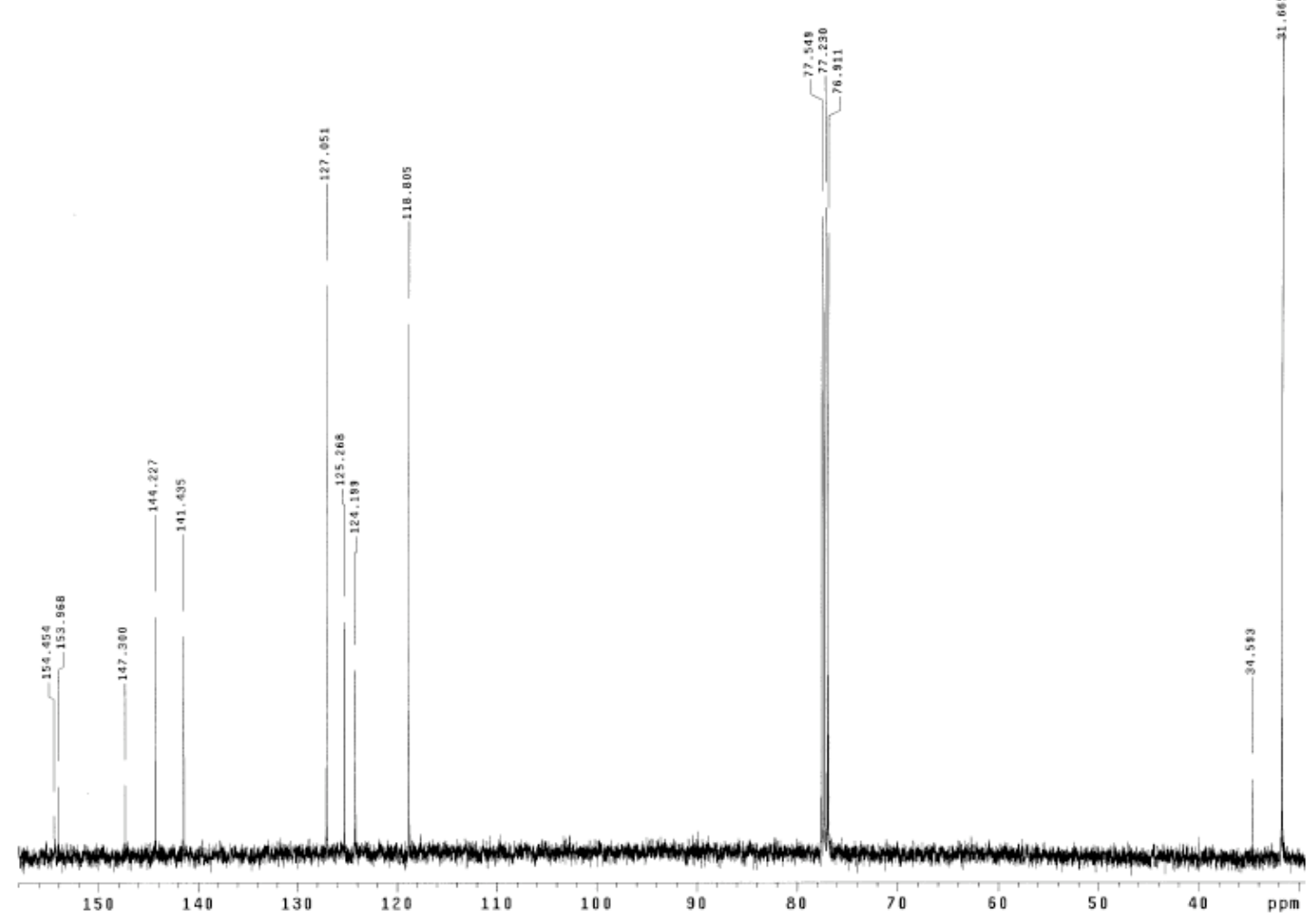

SI-16 

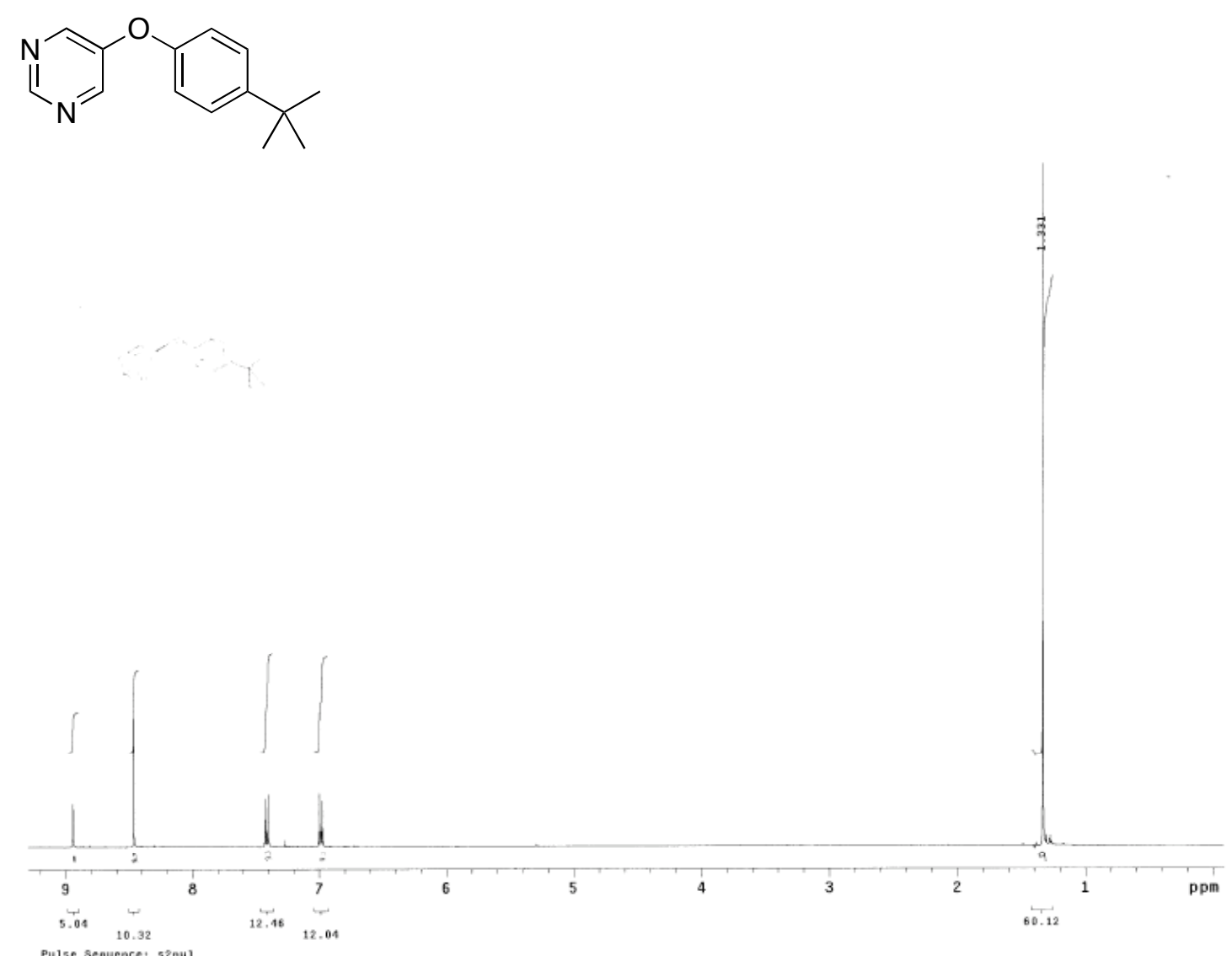
Pulse sequence: s2pul

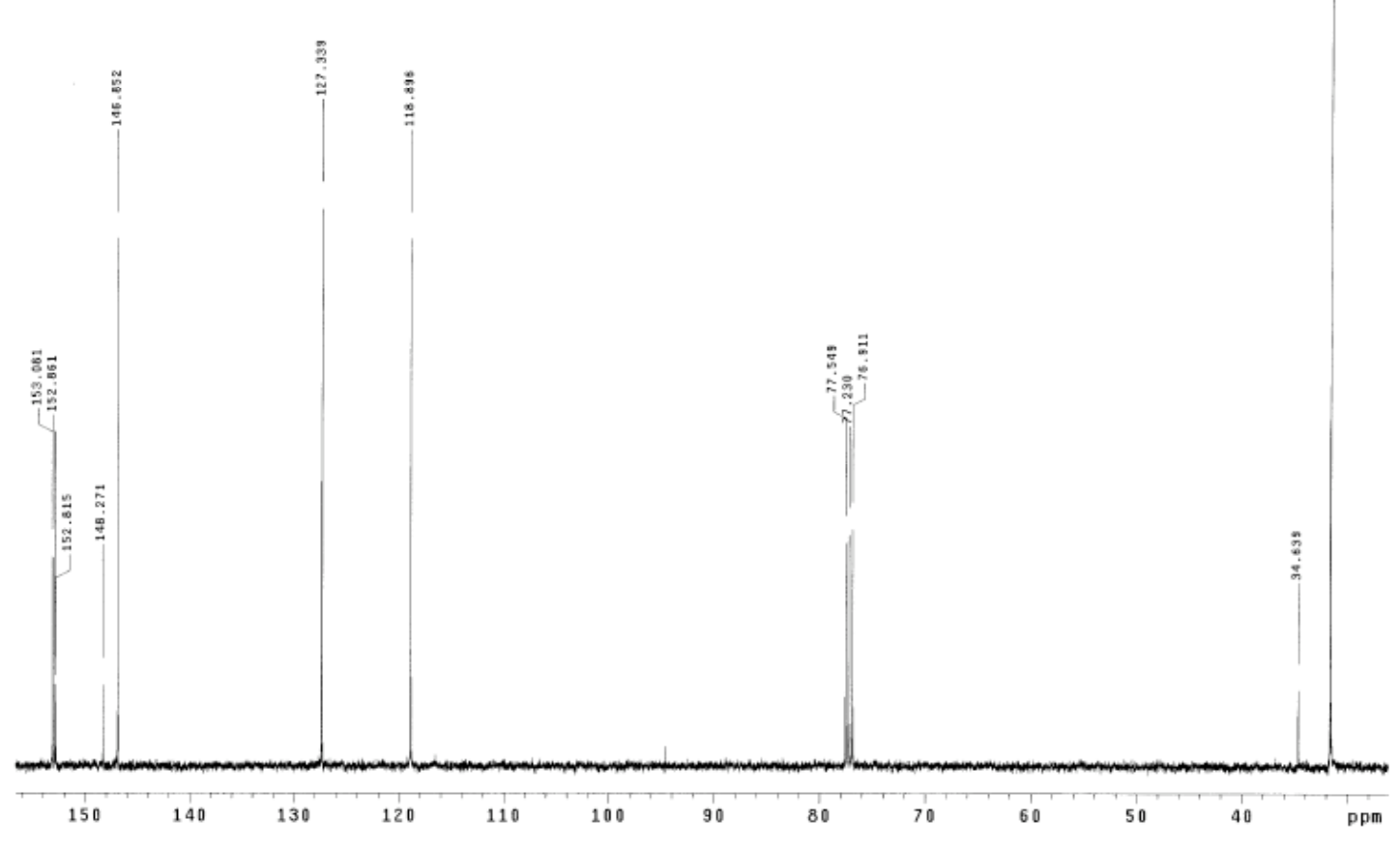

SI-17 
<smiles>CC(=O)c1ccc(Oc2ccc(C(C)(C)C)cc2)cc1</smiles>
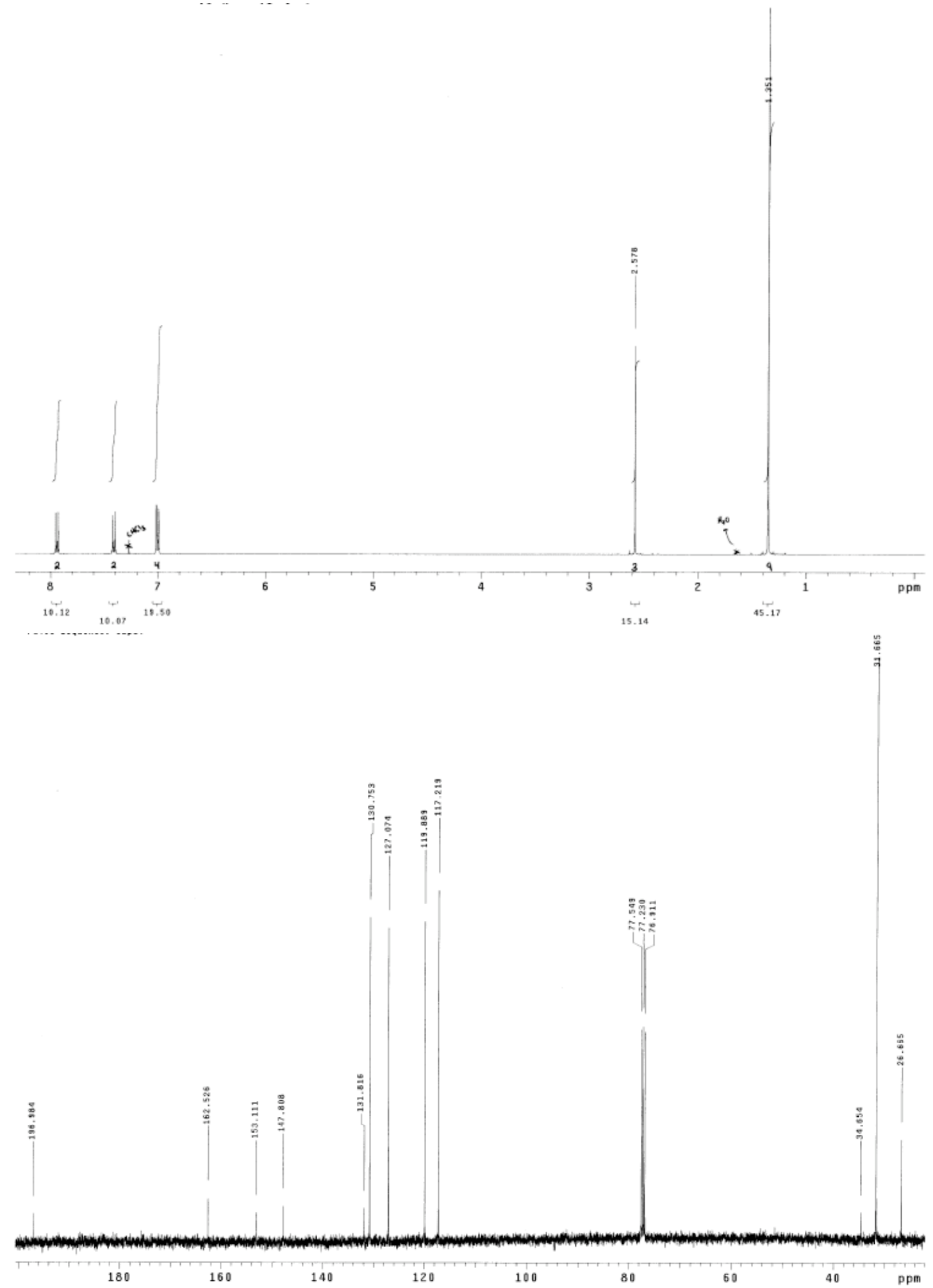

SI-18 

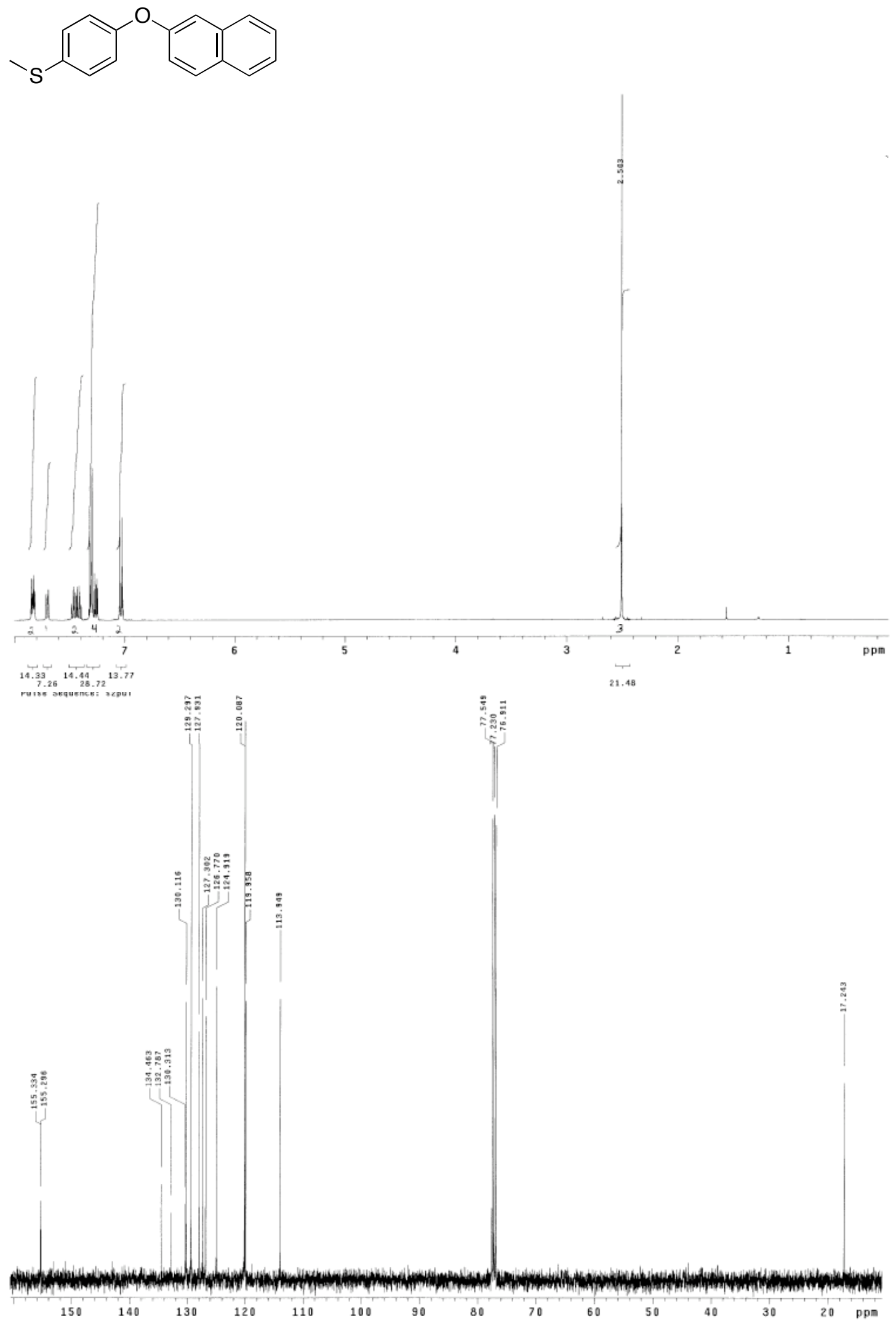

SI-19 

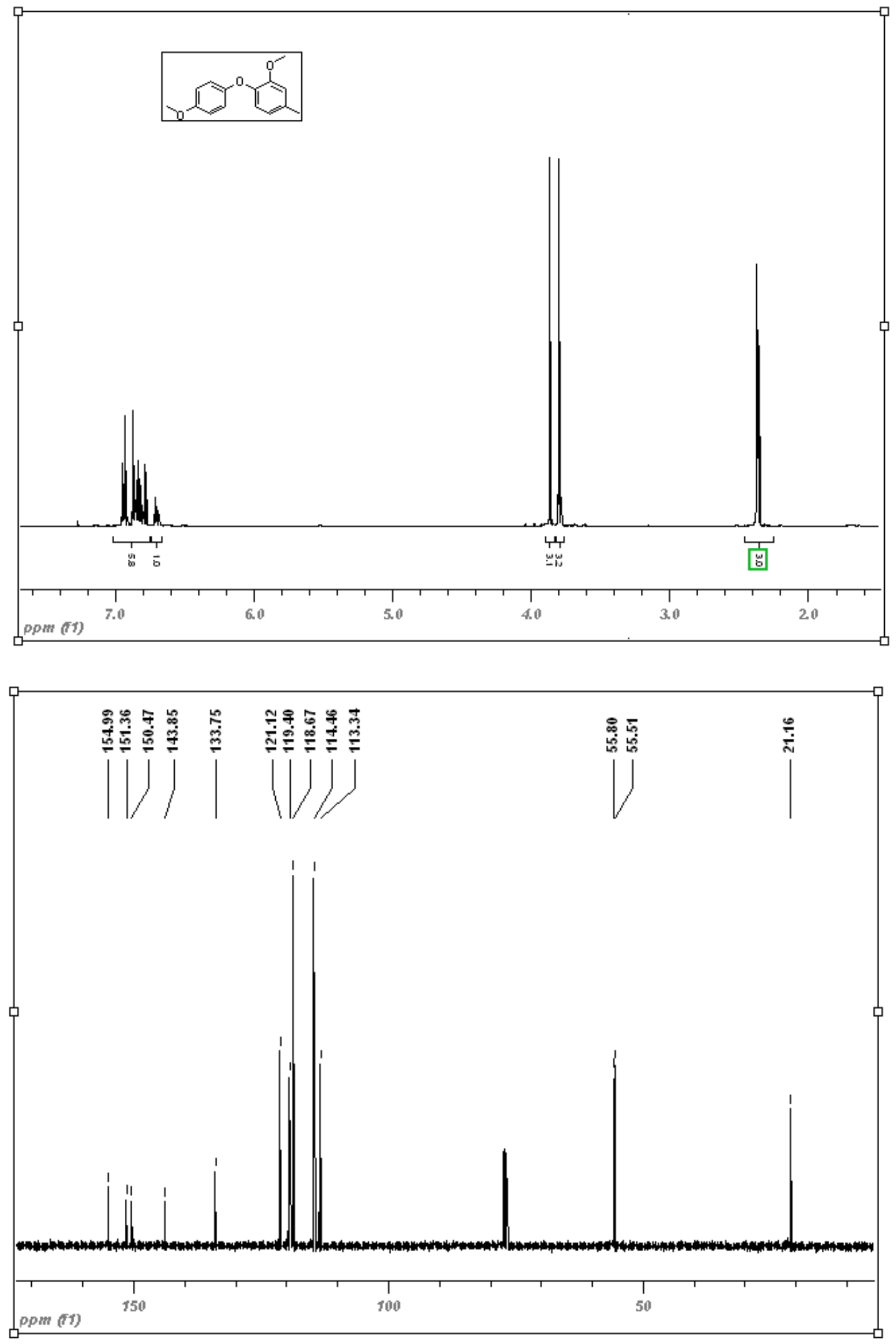

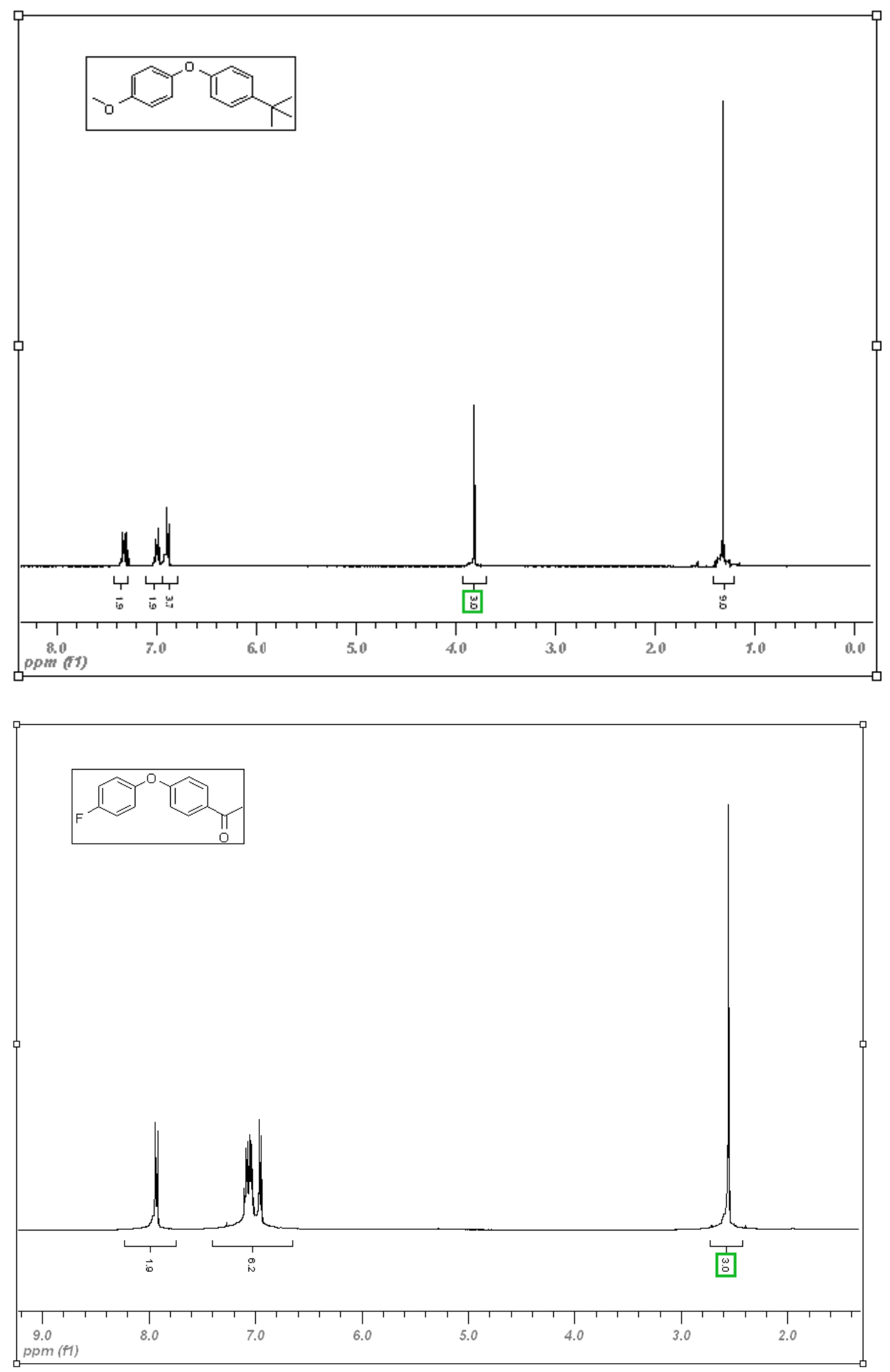

SI-7.1 


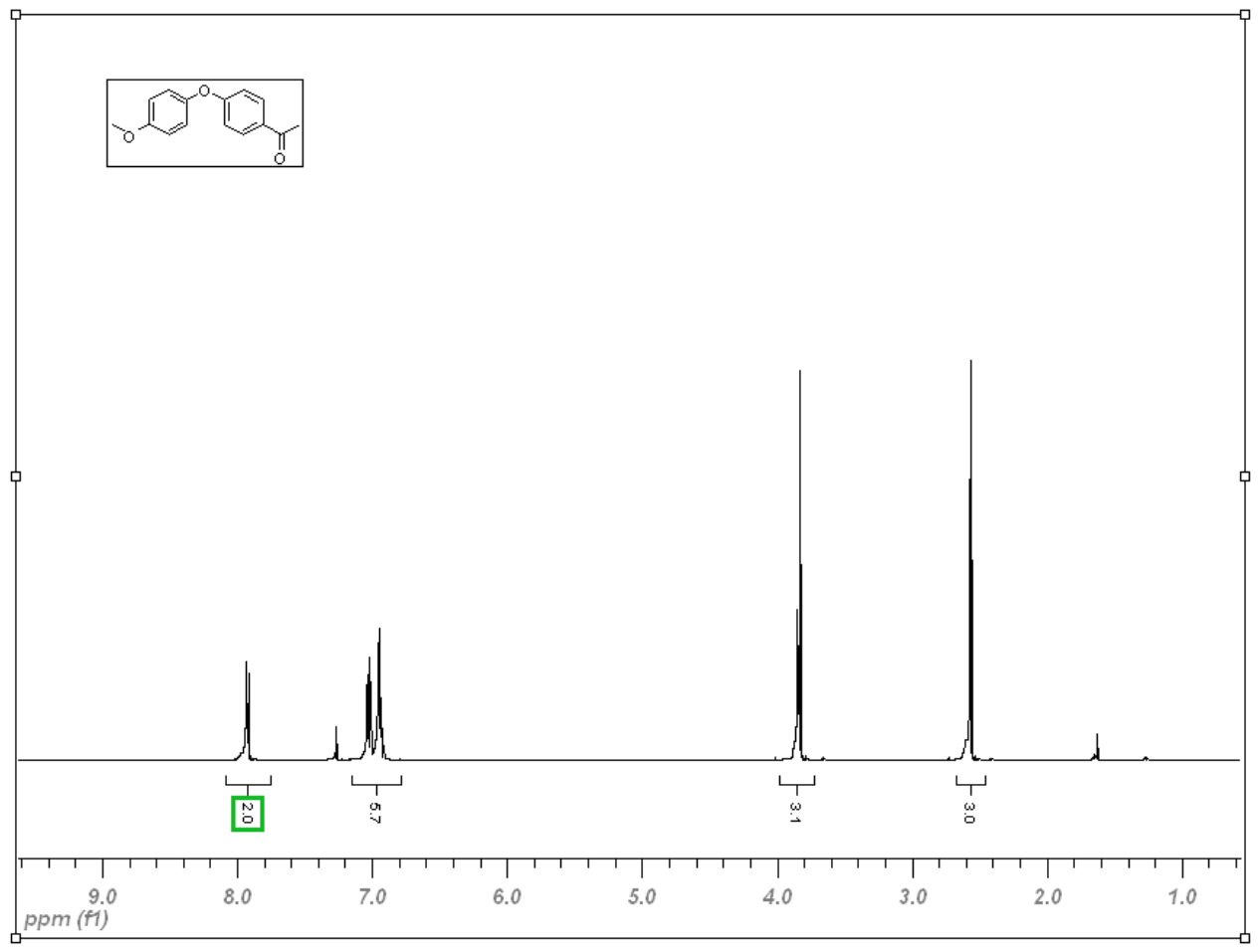

SI-7? 